\title{
A new journal: CEN Case Reports
}

The Japanese Society of Nephrology already publishes two official journals: Clinical and Experimental Nephrology (CEN) and the Japanese Journal of Nephrology (JJN). $C E N$ is published in English and is widely indexed. Recently, intense interest has been focused on education in clinical medicine, increasing the need for information on clinical issues such as case reports. Because of page limitations in $C E N$, we have decided to establish CEN Case Reports to facilitate publication of critical case reports and thereby contribute to clinical education.

On behalf of the Japanese Society of Nephrology, we eagerly look forward to your submissions.

Journal title: CEN Case Reports

Published format: Electronic online edition only (separate orders for print edition accepted)

Frequency of publication: twice a year (every 6 months) Contents: Case reports only

Initial publication: May 2012

Submission and publication cost: No charge for three or fewer printed pages, including color pages

Submission guidance: Use the online system Editorial Manager starting 1 September 2011.

For detailed instructions for submissions, please see the Instructions for Authors of CEN Case Reports.

http://www.springer.com/medicine/nephrology/journal/ 13730
[Inquiries]

CEN Case Reports, Editorial Office

c/o Springer Japan KK

No. 2 Funato Bldg. 1-11-11 Kudan-kita, Chiyoda-ku, Tokyo 102-0073, Japan

Tel.: +81-3-68317009

Fax: +81-3-68317010

E-mail: cencase@springer.jp

CEN Case Reports

Editor-in-Chief

Kenjiro Kimura, MD

Co-Editor-in-Chief

Tatsuo Sakai, MD

Deputy Editors

Toshiki Moriyama, MD

Shunya Uchida, MD 